\title{
Reevaluation of two models for judgments of perceptual intervals*
}

\author{
STANLEY J. RULE and DWIGHT W. CURTIS \\ University of Alberta, Edmonton, Alberta, Canada
}

\begin{abstract}
In Marks and Cain's (1972) experiment, 12 Ss estimated magnitudes of stimuli presented one at a time and differences in magnitudes of stimuli presented in pairs for heaviness of weight, roughness of emery papers, and area of circles. Their results appeared inconsistent with the two-stage model of magnitude estimation. But the expressions fitted to the data were different from those previously used in tests of the model. An additive constant was omitted. A reanalysis of their data with the constant included yielded support for the model for all three continua. An alternative model proposed by Marks and Cain was reasonably consistent with the data for weight, while inconsistent with the data for roughness and area.
\end{abstract}

The purposes of this paper are to reexamine some data which Marks and Cain (1972) have interpreted as inconsistent with the two-stage model of magnitude estimation and to examine further an alternative model for difference judgments proposed by Marks and Cain. With respect to the former purpose, it is argued that the functions fitted to their data differed from those employed in previous tests of the two-stage model, and therefore a conclusion that their data failed to replicate previous findings is uncertain.

It is assumed in the two-stage model that the relation between subjective magnitude $\left(\Psi_{0}\right)$ and the physical measure of the stimulus $(\phi)$ and the relation between subjective number $\left(\Psi_{\mathrm{N}}\right)$ and objective number $(\mathrm{N})$ are each power functions which may be expressed as

$$
\begin{aligned}
& \Psi_{\phi}=\mathrm{a} \phi^{\mathrm{k}}, \\
& \Psi_{\mathrm{N}}=\mathrm{cN}^{\mathrm{q}} .
\end{aligned}
$$

Setting $\Psi_{\phi}=\Psi_{\mathrm{N}}$ and combining Eqs. 1 and 2 yields the following relation between number and the physical stimulus

$$
\mathrm{N}=\mathrm{a}^{\prime} \phi^{\mathrm{km}},
$$

where $m=1 / q$ and $a^{\prime}=(a / c)^{m}$.

An additive constant, which represents a displacement from origin on the response scale, has been included in tests of the two-stage model (e.g., Curtis, Attneave, \& Harrington, 1968). When this constant is included, the relation between Ss' judgments, J, and the physical stimulus is expressed as

$$
\begin{aligned}
J & =N+b \\
& =a^{\prime} \phi^{k m}+b .
\end{aligned}
$$

The input and output exponents, $\mathrm{k}$ and $\mathrm{m}$, cannot be

*This research was supported in part by Grant AO 151 from the National Research Council of Canada. The authors wish to thank Lawrence E. Marks for making his data available. estimated separately by fitting Eq. 4 to magnitude estimation data. In previous studies, the expression fitted to magnitude estimation of single stimuli was the psychophysical relation

$$
J=a^{\prime} \phi^{n}+b .
$$

To test the two-stage hypothesis, the exponent $n$ has been compared with its predicted value from the product $\mathrm{k} \mathrm{x} \mathrm{m}$, obtained from another judgment task. In the Curtis et al study, estimates of $k$ and $m$ were obtained from judgments of the magnitude of differences between weights. Fitted to the difference judgments, $J_{i j}$ was an expression derived from the two-stage model,

$$
\mathbf{J}_{\mathrm{ij}}=\mathrm{a}\left(\phi_{\mathrm{i}}^{\mathrm{k}}-\phi_{\mathrm{j}}^{\mathrm{k}}\right)^{\mathrm{m}}+\mathrm{b} .
$$

For individual $\mathrm{Ss}$, the product of $\mathrm{k}$ and $\mathrm{m}$ obtained from difference judgments closely predicted the exponent $n$ from magnitude estimation of single stimuli.

In Marks and Cain's (1972) experiment, 12 Ss judged the magnitude of single stimuli and differences between stimuli from three continua: weight, roughness, and circle area. The expressions fitted to their data were

$$
\mathbf{J}=\mathrm{a} \phi^{\mathrm{n}},
$$

for single stimulus judgments, and

$$
\mathbf{J}_{\mathbf{i j}}=\mathrm{a}\left(\phi_{\mathrm{i}}^{\mathrm{k}}-\phi_{\mathrm{j}}^{\mathrm{k}}\right)^{\mathrm{m}},
$$

for difference judgments. They omitted the additive constant, b, in Eqs. 5 and 6 on the grounds that "there appears to be neither empirical nor theoretical justification for the addition of another parameter to be estimated [p. 15]." Although Marks and Cain disagreed with the inclusion of the additive constant, it is unfortunate on purely empirical grounds that they did not present an analysis with the constant included because their results may be interpreted as a failure to replicate findings from several tests of the two-stage model (Curtis, 1970; Curtis et al, 1968; Rule, Curtis, \& 


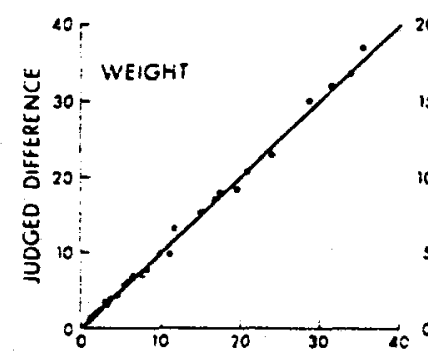

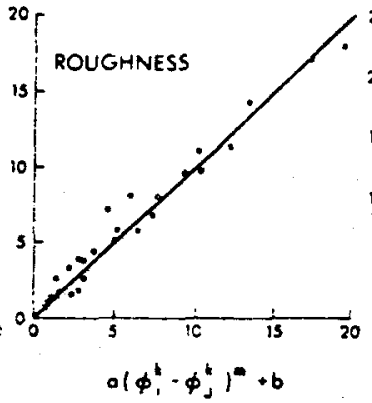

$o\left(\phi_{1}^{k}-\phi_{j}^{*}\right)^{*}+b$
Markley, 1970). An apparent failure to replicate results at another laboratory leaves both sets of data subject to doubt. However, the question arises as to whether Marks and Cain's failure to find support for the two-stage model was a consequence of responses inconsistent with those previously reported or merely a difference of opinion about a parameter to be estimated. We attempted to resolve the issue by fitting Eqs. 5 and 6 to Marks and Cain's data and by comparing the obtained exponent $\mathrm{n}$ with its predicted value from the product $\mathrm{kxm}$.

As an alternative to the two-stage model, Marks and Cain suggested that Ss' difference judgments were a compromise between perceptual intervals and perceptual ratios. The model tested was expressed as the following weighted sum of differences and ratios of magnitude estimates of single stimuli:

$$
J_{i j}=d\left[\left(J_{i}-J_{j}\right)+r \frac{J_{i}}{J_{j}}\right],
$$

where $J_{i}$ and $J_{j}$ denote magnitude estimates of the $i^{\text {th }}$ and $j^{\text {th }}$ stimuli. Plots of the observed difference judgments against their predicted values provided a reasonably good fit for judgments of weight, while data for roughness and area fitted less well.

Because response measures appear on both sides of Eq. 9, appropriate estimates of parameters are difficult and the only test of the model is provided by visual inspection of the data. These difficulties can be remedied by substituting the right side of Eq. 7 (the form of the psychophysical function preferred by Marks and Cain) for each $J_{i}$ in Eq. 9 to yield

$$
\mathrm{J}_{\mathrm{ij}}=\mathrm{d}^{\prime}\left[\left(\phi_{\mathrm{i}}^{\mathrm{n}}-\phi_{\mathrm{j}}^{\mathrm{n}}\right)+\mathrm{r}^{\prime}\left(\frac{\phi_{\mathrm{i}}}{\phi_{\mathrm{j}}}\right)^{\mathrm{n}}\right]
$$

where $d^{\prime}=$ ad and $r^{\prime}=r / a$. When Eq. 10 is fitted to difference judgments, an estimate of the exponent $n$ is obtained which may be compared with the exponent from magnitude estimation of single stimuli. This procedure provides a test of the difference-ratio hypothesis which is similar to that employed for testing the two-stage model.

\section{RESULTS}

The extent to which Marks and Cain's data replicate previous research on the two-stage model was evaluated by fitting Eq. 6 to geometric means of the Ss' estimates of differences in subjective magnitude and Eq. 5 to geometric means of magnitude estimates of single stimuli for weight, roughness, and area. The expressions were fitted to the data by a nonlinear least-squares solution applied to untransformed stimulus and response measures. In the solution, each squared deviation was weighted by $1 / \mathrm{J}^{2}$. This weighting strategy is similar to that provided by a linear least-squares solution applied to logarithmically transformed measures. A general description of the expression minimized has been presented by Rule et al (1970). Plots of difference judgments against the values predicted from fitting Eq. 6 to the data are presented in Fig. 1. Examination of the plots reveals that for each continuum, the expression provided a close approximation to the data. The percentages of the variance accounted for by the function were $99 \%$ for weight, $96 \%$ for roughness, and 99\% for area. Least-squares estimates of input and output exponents from Eq. 6 are presented in Table 1, along with the exponent, n, obtained from fitting Eq. 5 to magnitude estimation of single stimuli. The product $\mathrm{k} \mathrm{x} \mathrm{m}$ closely predicted the exponent, $\mathrm{n}$, for each of the three continua, a result consistent with the two-stage model.

To test the difference-ratio hypothesis, Eq. 10 was fitted to difference judgments. Plots of the observed values against those predicted by the function are presented in Fig. 2. The percentages of the variance accounted for by the function for weight, roughness, and area were $96 \%, 85 \%$, and $93 \%$, respectively. For each continuum, the fit of Eq. 10 was less satisfactory than the fit of Eq. 6 . The crucial test of the difference-ratio model, however, is how well the exponent from fitting Eq. 10 agrees with the exponent from fitting Eq. 7 to magnitude estimation of single stimuli. The estimates of $\mathrm{n}$ from difference judgments and magnitude estimation of single stimuli, respectively, were 1.15 and 1.11 for weight, 1.46 and 1.20 for roughness, and 1.08 and .75 for area The estimates of the exponent were reasonably close for weight but not 
for roughness or area. Therefore, the data do not provide general support for the difference-ratio model.

\section{DISCUSSION}

The present reanalysis revealed that Marks and Cain's data are not generally consistent with the difference-ratio hypothesis, but that they are consistent with the two-stage model of magnitude estimation. This result reconfirms, as a general empirical fact, previous findings that the product of input and output exponents ( $\mathrm{k} \mathrm{x} \mathrm{m}$ ) from difference judgments closely approximates the exponent $(\mathrm{n})$ from magnitude estimation of single stimuli. It should be noted in this regard that the data base for the two-stage model rests on experimental operations in addition to judgments of perceptual intervals or sums. Rule and Curtis (1973) devised a test of the two-stage model in which Ss compared the subjective magnitude of a weight with the magnitude expressed by a number. Power functions fitted to scale values obtained from a nonmetric scaling solution provided an input exponent for weight and an exponent for subjective number $(1 / \mathrm{m})$ which predicted the exponent for magnitude estimation of weight. Therefore, the findings are general for different experimental operations as well as for several different continua and different laboratories.

The primary difference in results from the present analysis, in which an additive constant was included, and those of Marks and Cain, in which the constant was not included, is the estimates of the output exponent, $\mathrm{m}$. Estimates of the output exponents differed by at least .31 (for weight) and as much as .48 (for area), while the input exponents, $\mathrm{k}$, differed between the two analyses by no more than .02 . Obviously, such large differences seriously affected the predicted exponent from the product of $\mathrm{k}$ and $\mathrm{m}$ in Marks and Cain's analysis. A similar result has been found for judgments of weight sums (Curtis \& Fox, 1969). These results indicate that, for the prediction from the two-stage model to hold, it may be necessary to include an additive constant in the expressions fitted to the data.

Marks and Cain have questioned the basis for a constant to correct for origin on the response scale. It is frequently assumed that a S's numerical responses are proportional to his subjective impression of stimulus
Table 1

Parameter Estimates of Magnitude and Difference Functions for Weight, Roughness, and Area

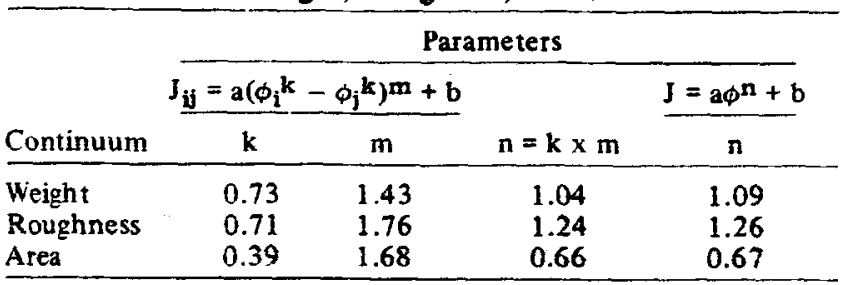

magnitudes, i.e., that $S s$ respond in accordance with the instructions. Obviously, a nonzero value of the additive constant is inconsistent with this assumed property of the S's response. However, tests of the two-stage model have indicated that Ss' numerical responses are not proportional to (or even linear with) subjective magnitudes, but that the relation is a power function. In view of this, it is not unreasonable to discard as well the assumption that the origin of the response scale necessarily corresponds to subjective zero.

As evidence against including a constant, Marks and Cain cited Stevens's (1969) reanalysis of Mashhour and Hosman's (1968) data from cross-modality matching. But, these results are not relevant to the issue. The constant employed by Mashhour and Hosman was introduced as a stimulus translation rather than as a response translation. Furthermore, Stevens's analysis with the constant omitted did not yield better agreement between predicted and observed exponents for cross-modality matching. The predicted values were improved by a "correction" for the regression effect, not by omitting the constant. There is, however, empirical support for a translation of the response scale. Ross and Di Lollo (1971) found that shifts in the stimulus series can produce changes in the origin of magnitude estimation scales such that scales are linearly related with a nonzero intercept. Further support was provided by Irwin and Corballis's (1968) study of magnitude estimation of loudness and softness of tones. They found that inclusion of the constant as a translation of the response yielded similar exponents with opposite signs, while its introduction as a translation on the stimulus scale (i.e., as a threshold parameter) did not. When neither constant was included, the psychophysical relations for both loudness and softness were curved on
Fig. 2. Difference judgments as a function of values predicted from the difference-ratio model (Eq. 10) for weight, roughness, and area.
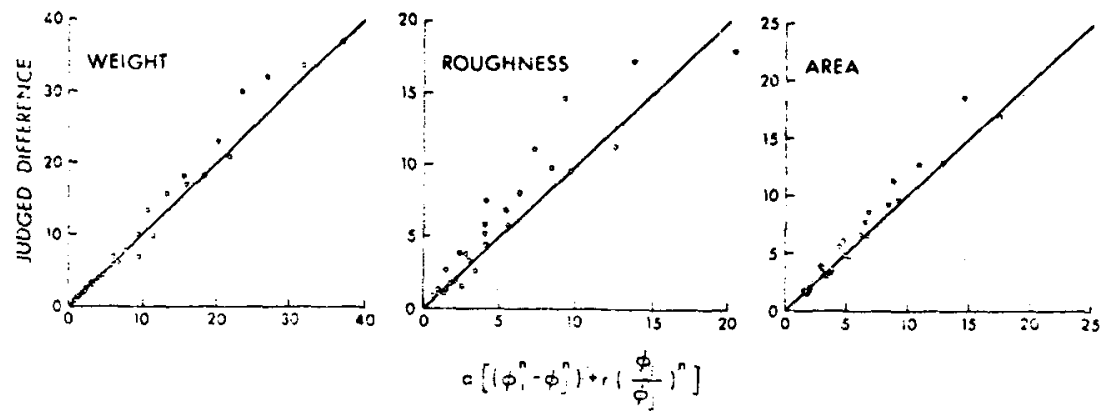
logarithmic coordinates. With respect to difference judgments, Rule et al (1970) found that a constant was required to describe the relation between judged difference and distance derived from nonmetric scaling. Because distance has a defined origin, the constant could be meaningfully introduced only as a translation on the response scale. It seems, therefore, that a correction for origin for magnitude estimation has a reasonable theoretical and empirical basis.

\section{REFERENCES}

Curtis. D. W. Magnitude estimations and category judgments of brightness and brightness intervals: A two-stage interpretation. Journal of Experimental Psychology, 1970, 83, 201-208.

Curtis. D. W.. Attneave, F., \& Harrington, T. L. A test of a two-stage model of magnitude judgment. Perception \& Psychophysics, 1968, 3, 25-31. -

Curtis. D. W., \& Fox. B. E. Direct quantitative judgments of sums and a two-stage model for psychophysical judgments. Perception \& Psychophysics, 1969, 5, 89-93.
Irwin, R. J.. \& Corballis. M. C. On the general form of Stevens' law for loudness and softness. Perception \& Psychophysics. 1968. 3, 137-143.

Marks, L. E., \& Cain. W. S. Perception of intervals and magnitudes for three prothetic continua. Journal of Experimental Psychology, 1972, 94. 6-17.

Mashhour, M.. \& Hosman, J. On the new "psychophysical law": A validation study. Perception \& Psychophysics, 1968, 3. 367-375.

Ross. J., \& Di Lollo, V. Judgment and response in magnitude estimation. Psychological Review, 1971. 78, 515-527.

Rule, S. J., \& Curtis, D. W. Conjoint scaling of subjective number and weight. Journal of Experimental. Psychology, 1973. 97, 305-309.

Rule, S. J.. Curtis, D. W., \& Markley, R. P. Input and output transformations from magnitude estimation. Journal of Experimental Psychology, 1970, 86, 343-349.

Stevens, S. S. On predicting exponents for cross-modality matches. Perception \& Psychophysics, 1969. 6, 251-256.

(Received for publication March 30, 1973; revision received June $11,1973$. ) 